H O S P I T A L I S M :

AND STATISTICS OF THE LATE OUTBREAK IN LINCOLN COUNTY HOSPTTAL.

By CHARLES BROOK, M.R.C.S. \&c., SURGHON TO THB HOSPITAL.

AFTER the refutation of the term " hospitalism" by such eminent men as have discussed the matter at the Clinical Society, my only excuse for using it lies in the fact that, whilst they have asserted the presence of pyæmia to be as general in private as in hospital practice, they have not attempted to prove that there does not at times exist, in some defective hospitals, a materies morbi which affects wounds or broken surfaces, and produces one or all of three infectious disorders-phagedæna, erysipelas, and pyæmia.

We know that some hospitals seldom or never suffer from these attacks, whilst others suffer very frequently; and surely there must be something connected with the hospitals themselves - faults either of construction, drainage, or management of wounds by either surgeons or nurses-to produce this result. I think therefore that Mr. Erichsen was justified in using the term "hospitalism" as applied to such cases engendered in defective buildings; but the canses of these outbreaks are, I believe, entirely avoidable, and therefore hospitalism should not exist.

Mr. Callender very justly disagrees with the general use of the term pyæmia, and certainly the pyæmia which we see in our hospital is very different in character from the socalled pyæmia in private practice. I can call to mind several cases of the latter in my own practice, and have had reason to welcome its advent: as in the case of a young married lady, who, on the second day after confinement, was seized with acute mania; this was checked by a large dose of opium (four grains) in the rectum ; pelvic cellulitis and tympanites ensued, with intense sensibility of the nervous system; for several days her life hung in the balance, but pain came in the upper arm, and she improved immediately, an abscess forming after a week or so. I think the local inflammation and deposit of pus saved my patient's life. I have at the present time the case of a young lady with typhoid fever; considerable lung complication in the early stage; the typhoid stage well marked for three weeks. Im. provement of general symptoms followed, but accompanied with frequent rigors and sweatings for a week, when she improved rapidly, and complained of acute pain in one knee. After a few days it became swollen; and now, with a cheerful countenance, moist tongue, and quiet pulse, the knee is going down, and her ankle is swelling. Here, again, the pyæmia came and relieved her just when I was despairing of her life. In these cases there was no septic poisoning, but a distinct febrile disorder, which was relieved by a deposit of pus; and if they are to be called pyæmia, they differ vastly from the last case of pyæmia occurring in my hospital practice, in which an old man with a broken leg, on which was a small chronic ulcer, occupied the same ward as a case of low erysipelas in a wound. After doing well for a fortnight the little ulcer looked angry; sickness, high temperature, and very pinched aspect suddenly came on; on the second day his knees swelled, and on the third day he died. Pus was found in his joints. There was at the time an outbreak of phagedæna and erysipelas in the hospital generally, an account of which is given below.

It appears to me that there are probably two forms of pyæmia. One arises from systemic infection caused by some poison perceptible only in its results, and generally fatal. This is almost peculiar to hospitals or crowded patients, and the same materies morbi which produces phagedæna or ery. sipelas in one patient may produce this form of pyæmia in another, as shown by Cases 1, 3, 4, in which the original outbreak in the hospital of phagedæna was followed by a case of acute pyæmia, and that by a case of erysipelas, all occurring in different wards. The other form of pyæmia arises from the absorption of matter within the body itself, relieving itself by abscesses or secondary deposits, and only proving fatal by exhaustion when deposited in joints or when very profuse. This second form is not affected by atmospheric conditions, and occurs in private as frequently as in hospital practice. It may be greatly avoided by careful management of wounds and general attention to the condition of the patient; and this is the sole secret of the success of my friend and teacher, Mr. Callender, his constant supervision, characteristic cleanliness, and minute attention to detail preventing the possibility of any fetid discharge being left for absorption. Given a hospital with perfect sanitary arrangements and this careful mode of managing wounds, and I firmly believe that we should hear little of any form of pyæmia. Mr. Jonathan Hutchinson's conclusion, that "ventilation is of no use at all," and that "erysipelas is frequent.]y caused by such so-called hygienic arrangements," must be a most dangerous theory for so great an authority to promulgate amongst the profession. And his conclusion that hospital air has very little to do with the causation of pyæmia, because he has seen many cases of this disease in the London Hospital and none in the Metropolitan Free Hospital, only strengthens the fact that some buildings are affected by hospitalism, while others remain free.

The following are the cases which occurred in the late outbreak in the Lincoln County Hospital in the months of November, December, and January last. The hospital was apparently in a healthy condition when the first case occurred, seven months having elapsed since the last outbreak. They are placed in the order in which they were attacked.

1. Female. No. 1 female ward. Housemaid's knee. Suppurated, was opened, and in a few days began to slough; phagedæna set in, and extended three parts round the joint; exhaustion very great. Strong nitric acid was applied; large doses of quinine did most good. Recovered in four months. She was housemaid in the hospital, but had nothing to do with the wards. The disease therefore broke out in a person who had been living in the hospital air.

2. Female. No. 1 female ward. Fatty tumour removed from back. Low form of cellulitis and great sloughing. Recovery in eight weeks.

3. Male. No. 1 male ward. Contusion over iliac glands. Counter-irritation employed; followed by rigors, sweats, swelling of knees and ankles. Rapid death. Pus found in the joints. This case was in the extremity of the building, furthest from the previous cases.

4. Male. No. 3 male ward. Contusion of elbow. Erysipelas; diffuse sloughing cellulitis; severe constitutional disturbance; rigors; very high temperature. Recovery in thirteen weeks.

5. Female. No. 3 female ward. Amputation of leg. Sloughing of flaps; rigors; secondary abscesses; very high temperature. Recovery in nine weeks.

6. Male. No. 2 male ward. Amputation of toe. Sloughing. Recovery.

7. Male. No. 3 male ward. Simple fracture of tibia and fibula. Small chronic ulcer, the size of a shilling. Sudden attack of vomiting; rigors; swelling of joints. Rapid death. Pus found in joints.

8. Male. No. 3 male ward. Periostitis. Two attacks of erysipelas. Recovery in seven weeks.

9. Male. No. 4 male ward. Small ulcer of leg. Erysipelas, with deep-seated sloughing and suppuration from glutei muscles to heel. Still in hospital.

10 \& 11. Males. No. 2 male ward. Ulcers of legs. Erysipelas. Recovery.

12 \& 13. Males. No. 5 male ward. Strumous abscesses. Erysipelas ; cellulitis. Recovery.

14. Male. No. 2 male ward. Diseased ankle. Erysipelas; sloughing. Recovery.

15. Male. No. 4 male ward. Removal of fatty tumour. Severe erysipelas. Recovery.

16. Male." No. 2 male ward. Cancer of lip. Removal. Diffuse erysipelas; extensive inflammation of larynx; tracheotomy. Death.

17. Male. No. 5 male ward. Fistula in ano. Erysipelas. Recovery.

18. Female. No. 2 female ward. Wound of arm. Erysipelas. Recovery.

19. Male. No. 2 male ward. Wound of finger. Erysipelas. Recovery.

In the months of November, December, and January, 48 cases of open wounds were admitted; 19 of these, or 40 per cent., suffered from either phagedæna, pyæmia, or erysipelas. The cases were distributed over all the wards, and broke 
out with the greatest irregularity, and the first case was that of a healthy person who had been for some time resident in the hospital,- - showing that the infection pervaded the entire building. Mr. Erichsen's outbreaks in University College Hospital appear to have been confined nearly to one ward, where operations accumulated. No operations were performed in the Lincoln Hospital from the appearance of the second case of phagedæna in November until February, except the case of tracheotomy, which proved fatal. By not admitting chronic ulcers-a most noxious affection,-and by diminishing the number of patients, we have now got rid of this state of things for a time.

Lincoln.

\section{CASE OF CIRSOID ANEURISM TREATED BY INJECTION OF PERCHLORIDE OF IRON ; DEATH FROM EMBOLISM.}

BY JAMES F. WEST, F.R.C.S., SEMIOR SURGEON TO THE QUREN'S HOSPITAL, BIRMIMGHAY.

Despite the brilliant success which has attended the employment of coagulating injections for the treatment of nævus in the hands of MM. Broca, Gosselin, Demarquay, Professor Pitha, and others, they are evidently dangerous remedies in certain cases, especially when the tumours are about the face and head; and it is incumbent on those who have had fatal cases to publish them as a warning to others not to use such injections in the future, but to adopt some less hazardous mode of treatment. Mr. Bryant* mentions that he lost a patient from embolism a few minutes after injecting a nævus, though he does not say on what part of the body it was situated. Mr. Thomas Smith, in an excellent paper on the same subject, gives several cases in which a fatal result attended the use of perchloride-of-iron injec-

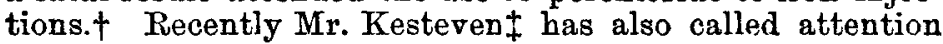
to an unsuccessful case in his practice; and although he attributes death in that instance to "spasm of the glottis, induced by mental emotion," I cannot help thinking the result was more likely due to embolism. The absence of a post-mortem examination, however, prevents the solution of the question.

Such experience as is afforded by these records and by the following case must make ail thoughtful surgeons pause before recommending the injection plan of treatment, and induce them rather to have recourse either to enucleation, the galvano-cautery, the ligature, or, what I very much prefer, the wire écraseur. In ThE LaNCET of March 4th, 1871, I have reported several cases in which considerable næroid growths were treated by me in this manner with success; and further experience has shown me that the écraseur is one of the best, if not the best means we possess for the removal of vascular tumours.

Alfred G. T_ aged nine months, was admitted into the Queen's Hospital, under my care, on June 2nd, 1873, with a nævoid tumour, about the size of a small marble, on the right ala nasi, near the tip. It had existed from birth, but was slowly growing, and beginning to involve the left ala. It was soft and compressible, and over its surface large and distended capillaries were seen in three or four spots, each as large as a pea. Deformity from the prominent situation of the tumour necessitated operative interference, and as it was considered that no mode of operation offered so good an opportunity for the prevention of an ugly cicatrix as the injection of solution of perchloride of iron, it was decided to use it. At 11 A.M., cnloroform having been given, three drops were injected by a hypodermic syringe into the lowest part of the tumonr. The child struggled and cried, but there was no other unfavourable symptom. Three more drops were then injected into the upper part of the tumour, and three at another highly vascular spot, when, on withdrawing the syringe, the child's face changed suddenly to a dusky bue, the hands and feet became blue, the pulse could not be felt, and the breathing seemed to be arrested; on raising the eyelids, the eyeballs were seen to be fixed and turned upwards and outwards, and the pupils were slightly

* Practice of Surgery, $p .204$

† ThI LANCET, Aug. 17th, 1867. dilated. The tongue was at once drawn forwards, and artificial respiration kept up for twenty minutes. During some part of this time the child breathed naturally, and then would cease to breathe until Silvester's method was again used. Until 6 o'clock the same evening he continued in a half-unconscious state, with a very feeble pulse, dilated pupils, livid bands and feet, and the angle of the mouth on the right side somewhat drawn down. About every ten or fifteen minutes he would start crying, and then relapse into his former semi-comatose state. During the evening the extremities became warmer and of a more natural colour the breathing less laboured, and the pulse better; but the eyeballs rolled about, and the child could not be roused to take notice, and could hardly be induced to imbibe milk from his bottle. He continued in this state for three days, when be died, with well-marked symptoms of hemiplegia.

At the post-mortem examination, the membranes of the brain were found slightly congested; the anterior portion of both lobes of the cerebrum, and also the inner surface of the brain as seen in the walls of the lateral ventricles, were reduced to a soft pulp, while the posterior part of the brainsubstance was in a healthy state. A clot was seen stretching from the right internal carotid artery along the middle cerebral artery of the same side. The lungs were congested. The heart contained a small quantity of dark fluid blood. The liver, kidneys, and other viscera were normal.*

That embolism was produced in this instance by the injection of the perchloride of iron does not admit of a doubt, and I therefore unhesitatingly confirm Mr. Thomas Smith's opinion that we are justified in rejecting it as a remedy for nævi on the face.

Birmingham.

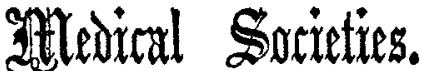

\section{PATHOLOGICAL SOCIETY OF LONDON.}

Tumsday, MaRCH 17Th, 1874.

Sir Wir. Jenner, President, in the Chatr.

\section{DISCUSSION ON CANCER.}

Sir JAMme PAget. - I regret that $I$ was unable to be present at the last meeting of the Society, but I have had the advantage of having been able to read the careful report of Mr. De Morgan's speech at the opening discussion, published in the journals, and I probably find therein a very much more careful agreement between myself and him in reference to the pathology of cancer than I might have supposed if I had only heard the address delivered and had seen the preponderance, to all appearance, though not in reality, of the view which he held of what may be generally understood as its local origin; for I find that $\mathrm{Mr}$. De Morgan holds as I do-and not I alone, but I think the large body of those engaged in the study of pathology and of surgical practice too-that we must look at the same time for a local and a constitutional origin of cancer. If Mr. De Morgan will allow me, I will read one or two sentences of his own on the matter, that they may serve as a warning to myself lest I should diverge too far. $\mathrm{He}$ says: "All, in fact, that we see in the life of cancer naturally leads to the belief that the disease must from the first be more than a mere local tissue change; and in one sense, and to a certain extent, I must admit that this belief is well founded." And again: "The view which I would maintain is that, though local in its origin, there is in some, possibly in all, cases a predisposition to the disease which may possibly be distributed through the system, but much more probably has its seat in some among the tissues of the body." To this doctrine I should entirely conform; and if I could find anything to discuss or anything in $\mathrm{Mr}$. De Morgan's address to which I could object, it would be, not in a doctrine such as this, but rather in what I may call the only half-willing manner in which he seems to admit it-the manner in which he seems disposed to de-

* The notes of the case and of the post-mortem were kindly furnished me by Mr. Gilbert Smith, the house-surgeon. 